In patients with hypertensive heart failure an effective fall of blood-pressure is desirable. Reserpine in a dose of $2.5 \mathrm{mg}$. by mouth or by intramuscular injection will lower the bloodpressure smoothly. More powerful hypotensive drugs have little place in the treatment of the young adult African because only exceptionally is there adequate nursing staff, and after the crisis is over the patient may live far away from the follow-up clinic, even if he could afford to buy the expensive drugs which he needs.

Unless associated or complicating diseases are tackled as soon as emergency treatment is over they may prevent the patient from getting home and back to work quickly, which for the labourer may mean the end of his job.

\section{Systemic Oedema}

Digoxin and a diuretic are the sheet anchors of treatment. The dose of digoxin is similar to that described above, but there is no need to give the first dose of $1 \mathrm{mg}$. intravenously unless there is atrial fibrillation with a rapid ventricular rate. Mersalyl is again ideal as a diuretic, but for treatment out of hospital an oral diuretic such as hydrochlorothiazide $50 \mathrm{mg}$. will be easier to manage. It should be given often enough to prevent oedema forming: the dose will vary from $50 \mathrm{mg}$. weekly to $50 \mathrm{mg}$. daily. If possible, potassium chloride $1 \mathrm{~g}$. three times a day should be taken on days when the diuretic is taken. One of the least palatable measures, which is often necessary in the very oedematous patient, is to restrict salt in his food. When relatives bring food to him in hospital this may prove almost impossible, but it is well worth trying to stop his family putting salt into the pot or giving him foods rich in sodium. When there is much ascites, which is very common in the African with cardiac failure, it should not be tapped unless the abdomen is very tense: instead, diuretics and a diet without added salt should be tried first.

\section{Summary}

The young adult African is of ten seen with advanced cardiac failure.

When pulmonary oedema overshadows all other signs a functional diagnosis only is possible. Some of the common causes of pulmonary oedema in the African are mentioned and their diagnosis is discussed.

Patients with cardiac failure and much systemic oedema can be confused with those in whom oedema is due to other causes, for example hypoalbuminaemia. Problems in the diagnosis of these patients are discussed.

Treatment is urgent in cases of pulmonary oedema. Followup treatment is governed as much by the patient's means and the distance of his home from hospital as it is by his disease.

I am grateful to the Wellcome trust for the support of my work in Ethiopia.

\title{
DISEASES OF THE SKIN
}

\section{Management of Disorders of the Nails}

\author{
PETER D. SAMMAN,* M.D., F.R.C.P.
}

The management of nail disorders is far from satisfactory at the present time. Very little is known about the pathology of these conditions, and though most nail deformities are of little importance from a functional point of view they are of great cosmetic importance.

There are three nail diseases which are often seen by the dermatologist: chronic paronychia, fungal infections, and psoriasis. They are very often misdiagnosed. In addition, patients present with one of a number of nail symptoms, the causes of which are often quite unknown, and treatment for which must therefore be empirical.

\section{Chronic Paronychia}

Chronic paronychia is quite the most common nail disorder for which the patient seeks advice. It is a disease mainly of women, and of women who have their hands constantly in water. It embraces, therefore, housewives (and all others who do much housework), bar tenders, laundry hands, nurses, and many others. It is more likely to affect people who suffer from cold hands. The characteristic features are the loss of the cuticle, bolstering and discomfort of the posterior nail fold, and the appearance of occasional beads of pus in the groove at the nail base opened up by the loss of the cuticle. From time to time a more acute inflammation may * Physician, Dermatological Department, Westminster Hospital, London
S.W.1, and St. John's Hospital for Diseases of the Skin, London W.C.2. occur for a few days. Nail deformity appears later and most often consists of roughness and discoloration of one edge of the nail. Less often the nail becomes cross-ridged, and in long-standing cases there may be greater deformity. Occasionally the nail may be reduced in size, a feature which is exaggerated by the great bolstering which may by now completely surround all but the free edge of the nail.

The aetiological factors in chronic paronychia are not universally agreed, but it is certainly not a true fungal infection. Monilia, usually Candida albicans, can be recovered from the nail fold in almost every case, ${ }^{1}$ but bacteria also play a part, especially in the more acute exacerbations. On culture a variety of organisms may be found, including Pseudomonas aeruginosa, which is probably responsible for the discoloration at the edge of the nail. ${ }^{2}$

From what has already been said it is obvious that griseofulvin can play no part in the treatment of this disorder. By far the most important part of treatment is to keep the hands dry. Unfortunately, this is often not possible, and treatment accordingly is not successful. The patient should be advised to wear rubber gloves for all wet work, preferably with thin cotton gloves under the rubber. Local applications are of less importance. In the early stages nystatin ointment is often the most useful preparation, and it should be applied frequently to the groove between the nail and the nail fold. Later, greater benefit may be obtained from the use of $15 \%$ sulphacetamide in $50 \%$ surgical spirit or $2-4 \%$ thymol in chloroform. Both these preparations help to dry out the nail 
fold, while $15 \%$ sulphacetamide is lethal to candida and to most probable bacterial contaminants. ${ }^{3}$ Treatment should not be considered complete until the cuticle has regrown. Many patients become exasperated with the slow progress, and request that the posterior nail fold be incised or the nail removed. These requests should be resisted, as they seldom give any permanent benefit.

\section{Fungal Infections}

Fungal infection of the nails is the second nail disorder frequently seen by the dermatologist, followed closely in incidence by psoriasis. These two disorders are often mistaken for one another, and chronic paronychia is often taken for a fungal infection.' In the early stages fungal infection may be limited to the edge of the nail, and closely resembles the nail in chronic paronychia, but the bolstering and loss of cuticle so characteristic of chronic paronychia is lacking. Later the whole nail plate may become discoloured yellow or brown, or less often chalky; it may become rough and soft, so that pieces break away. There is usually an accompanying fungal infection of the adjacent skin, and the nail bed may be thickened and discoloured. The diagnosis is established by finding filaments of fungus in full-thickness clippings from the affected nails after the clippings have been soaked in a few drops of $5 \%$ potassium hydroxide for 24-48 hours. Toe-nail infections are often seen in association with tinea pedis, but infections of the finger-nails are much less common. The one striking improvement in the treatment of nail conditions in recent years has been the use of griseofulvin in finger-nail infections. Most cases respond provided the dose is large enough and the patient perseveres with treatment. In the early stages a large dose is recommended, eight 125-mg. tablets a day, in divided doses for the first two weeks, for an adult male $140 \mathrm{lb}$. (63.5 kg.) or over, then reducing to six a day for about four weeks, and finally to four a day until the infected nails grow out satisfactorily. This usually occurs five to six months from the start of treatment. In women the starting dose will be six a day for a month, and then four a day, and for children three a day throughout will probably be sufficient. The drug is well tolerated in many cases, but if for any reason treatment has to be discontinued before the diseased area has grown out the result is unlikely to be satisfactory. The tablets should be crushed and not swallowed whole.

Toe-nail infections respond much less readily, and the slower rate of growth of toe-nails is certainly not the only reason for this. In the great majority of patients no treatment for toe-nail infections should be offered, but if the patient is young, or only one or a few nails are infected, these may be removed surgically and griseofulvin administered for a time during regrowth of the nail. Unfortunately, even in these cases some nails may quickly become reinfected.

\section{Psoriasis}

Psoriasis is probably the most widespread of all nail disorders but is usually a minor part of the disease, so that the patient seeks advice on account of the skin rather than the nails. In these cases diagnosis should be easy. It is only when there is no, or very little, psoriasis elsewhere that diagnosis may be difficult, especially as nail psoriasis may take several forms. In a minority of cases it can mimic a fungal infection very closely, but clippings from the nails will not reveal fungal filaments on microscopic examination.

Unfortunately, the management of nail psoriasis is very unsatisfactory. Some cases improve spontaneously, and some clear when the associated skin lesions are treated, but in many cases the nail lesions persist, unaffected by other treatment. In these resistant cases it is worth trying one of the fluorinated topical steroid applications, applied over the finger-tips and occluded each night for a time with polyethylene gloves. The nails may grow out normally under this treatment. Superficial $x$-ray therapy is occasionally helpful, but in many cases has no apparent value, and should therefore be used only if other methods fail.

\section{Nail Symptoms}

Two common nail symptoms are splitting and loosening. The latter term usually implies separation of the nail from its bed (onycholysis).

Nails may split into layers (lamellar dystrophy), or the free edge may split in its full thickness, usually along ridges. Splitting into layers is very common and is probably due to repeated minor traumata, especially the constant uptake and drying out of water. It happens more in winter than in summer, and as a complaint is almost confined to women. Treatment is not very satisfactory. Most cosmetic nail hardeners are of little value, but those which artificially add thickness to the tip of the nail may be helpful in some cases. One containing free formalin certainly hardens the nail, but may cause separation from the bed, especially, if used injudiciously. Sometimes nail varnish appears to be a factor in causation, and if this is suspected the patient should be advised to try omitting the use of varnish entirely for two or three months to assess its effect. If there is no improvement in this time the use of varnish should be restarted, as it does to some extent cover up the defect. In all cases the patient should be advised to keep her hands as dry as possible, and to use a hand cream containing glycerin each night.

Splitting in the length of the nail is less frequent, and is often associated with excess ridging or thinning of the nail. The patient should be examined for evidence of anaemia or impaired peripheral circulation, and these conditions should be treated if present. The nails should be kept cut as short as possible to prevent the cracks penetrating more deeply, and a glycerin hand cream used each night.

Onycholysis is a very prevalent symptom, and although many cases occur in perfectly healthy persons it may also be evidence of systemic disease, especially impaired peripheral circulation and thyroid disorders. It may also occur in many trades where the nails are particularly subject to trauma. In itself the condition is no more than a cosmetic blemish, but the loosened nail is very subject to trauma, which may cause pain and further separation. Moreover infection, often silent, is very apt to occur in the moist groove which is established. If the infection is due to Pseudomonas aeruginosa the nail may quite suddenly go black or blue-black and be accompanied by malodour. The patient should be advised to keep the affected nails cut as short as possible, and to keep them as dry as possible. One of a number of preparations may be used below the nail to try to prevent infection occurring while the nail has a chance to reattach itself to the nail bed. Attempts to clean out debris from below the nail should be discouraged. The preparations of most value are sulphacetamide $15 \%$ in $50 \%$ spirit, $2 \%$ thymol in chloroform, and a topical steroid application containing antibiotics and nystatin (Triadcortyl).

Ingrowing nails and onychogryphosis are not discussed here, because patients with these conditions are usually referred to the chiropodist or surgeon rather than the dermatologist.

\section{REFERENCES}

1 Marten, R. H., Brit. F. Derm., 1959, 71, 422 .

3 Ray, L. F., Arch. Derm. Syph. (Chic.), 1963, 88, 181. 